\section{The Titanium Oxide Bands.}

The fine structure analysis of the blue-green titanium bands, started nearly two years ago (see Phys. Rev., 29, 212; 1927, abstract 26), has now reached the point where it is possible to draw certain definite conclusions regarding the molecule responsible for the radiation of these bands. These conclusions are based on a complete verification of the combination principle, using some 1500 lines measured in the three bands $0-0,1-0$, and $0-1$ ( $\lambda \lambda$ 5167, 4955, and 5448 ).

Each band consists of three $R$ and three $P$ branches. These six series are nearly, but not exactly, parallel, so that they are continually crossing and re-crossing one another. Due to this fact, almost a majority of all measured lines are blends, and this has made both the empirical and the theoretical analysis unusually difficult. Good values for the triple origin have not as yet been obtained, but the three heads of the 0.0 band. lie at approximately 19349,19347 , and $19340 \mathrm{~cm} .^{-1}$. These will be denoted as the $a, b$, and $c$ heads respectively. In the case of the $P_{a}$ and $R_{a}$ branches, the resulting rotational energy functions fulfil quantitatively the expected relations between rotational and vibrational energy constants, and accordingly one can obtain from these functions very trustworthy values of the moment of inertia. The functions for the other two members of the triplet exhibit small positive and negative deviations respectively from the expected theoretical relations, and these deviations have not yet been satisfactorily explained.

There is no doubt that this band system is due to an oxide of titanium, and our earlier vibrational analysis (loc. cit.) showed that it is due to a diatomic molecule. The new additional fact that the individual bands have a triplet structure is practically certain proof that they are due to a molecule with an even number of electrons. Hence they must be due to neutral $\mathrm{TiO}$ rather than to $\mathrm{TiO}^{+}$.

It is not possible to carry any of the series within about twenty lines of the origin, due to the close spacing and overlapping of all series in that region. Hence one cannot say definitely that no $Q$ branches are present. But if they occur, they must be very short and weak. It is therefore very probable that these bands are due to a ${ }^{3} P-{ }^{3} P$ transition (like the Swan bands), rather than to ${ }^{3} P-{ }^{3} S$, or ${ }^{3} P-1 S$. If TiO is similar to CO, the normal level should be ${ }^{1} S$, and on that assumption the lower level of these titanium bands is an excited level of the TiO molecule.

The values of the moments of inertia for the upper and lower levels are $(56 \cdot 76 \pm 0.03) \times 10^{-40}$ and $(51 \cdot 87 \pm 0.03) \times 10^{-40}$ respectively, corresponding to a nuclear separation $\left(r_{o}\right)$ of $1.694 \times 10^{-8} \mathrm{~cm}$. and $1.619 \times 10^{-8} \mathrm{~cm}$. The values of $r_{0}$ are approximately the same as those found for $\mathrm{AlO}$, and are some 15 per cent smaller than had been anticipated for TiO, from a study of the constants of other similar molecules. Hence the TiO molecule seems to be especially firmly bound.

The two band systems of possibly major import. ance in astrophysics are the Swan bands, found in $\boldsymbol{R}$. and $N$-type stars, and the titanium bands, found in $M$-type stars. The great preponderance of evidence is now that the Swan bands are due to a ${ }^{3} P-{ }^{3} P$ transition in the neutral $\mathrm{C}_{2}$ molecule, while the above facts indicate that the blue-green titanium bands are due to ${ }^{3} P-{ }^{3} P$ transition in the neutral $\mathrm{TiO}$ molecule. These two band systems, as is well known, never occur together in stellar spectra, a fact usually explained by assuming that one carrier exists in a reducing atmosphere, and the other in an oxidising atmosphere. The above conclusions are in agreement with this assumption.

A. Christy.

University of California, R. T. BIRGE. July 7.

\section{Two Lecture Demonstrations in Physics.}

(1) Two conducting wires or rods about five feet in length are stretched in a wedge shape, the gap being one-half inch at the bottom and three-quarters of an inch at the top. The upper three feet of the wires are covered with a glass tube. It is well known that if an induction coil is connected to the two wires, the resultant sparks will be carried up the wires by the rising ions; hence the spark will seem to climb the wires. When the spark reaches the top of the glass tube, the ions go out of the end, so that the spark begins again at the bottom. If now the top of the glass tube is tightly corked, the spark when it enters the tube will travel more slowly than along the lower part of the wires, and when it reaches the cork, a layer of ions will stay there, so that the spark, instead of returning to the bottom, will remain just under the

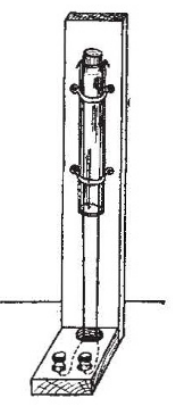

FIG. 1. cork. With the cork removed, an air blast down the tube will either prevent the spark from climbing or will blow it down the wires.

(2) Gray, in his book "Gyrostatics and Rotational Motion," has described a top which when rocked will walk in one direction along two parallel wires. When the top reaches the end of the wires, it is necessary to slide it back to its first position. If, however, the top is unbalanced with a small mass $M$, there will be an average upward force during each half revolution of the top of value $2 M v^{2} / \pi r$. This will lessen the

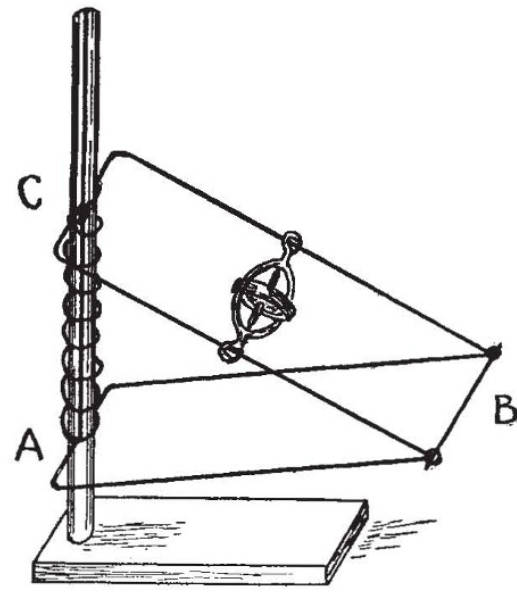

FIG. 2.

friction between the wires and the top, so that the top will slide backwards on the wires, provided the wires are exactly horizontal. We have shown before that the top will walk down a pair of inclined wires, since friction on the wires will start and stop precession. Therefore a top may be rigged up on inclined wires as shown in Fig. 2. With the spring extended so that $C$ is well above $B$, the top will walk from $C$ to $B$. With the spring depressed so that $C$ is slightly below $B$, the unbalanced top will slide back 